\title{
Abstract Analysis of Symbolic Executions
}

\author{
Aws Albarghouthi ${ }^{1}$, Arie Gurfinkel ${ }^{2}, \mathrm{Ou} \mathrm{Wei}^{1,3}$, and Marsha Chechik ${ }^{1}$ \\ 1 Department of Computer Science, University of Toronto, Canada \\ 2 Software Engineering Institute, Carnegie Mellon University, USA \\ ${ }^{3}$ Nanjing University of Aeronautics and Astronautics, China
}

\begin{abstract}
Multicore technology has moved concurrent programming to the forefront of computer science. In this paper, we look at the problem of reasoning about concurrent systems with infinite data domains and non-deterministic input, and develop a method for verification and falsification of safety properties of such systems. Novel characteristics of this method are (a) constructing under-approximating models via symbolic execution with abstract matching and (b) proving safety using underapproximating models.
\end{abstract}

\section{Introduction}

Concurrency has moved to the forefront of computer science due to the fact that future speedups of software rely on exploiting concurrent executions on multiple processor cores. Thus, the problem of creating correct concurrent programs is now paramount. Reasoning about such programs, i.e., determining whether properties of interest hold or fail in them, has always been difficult, especially if we consider "realistic" programs with infinite data domains (i.e., integer variables) and non-deterministic input. An example of such a program is the simple two-process mutual exclusion protocol shown in Fig. 1, where integer variables $x$ and $y$ are set non-deterministically (see Section 2 for more detail).

Approaches to reason about concurrent systems can be split into four categories. (1) "Classical" model-checking techniques, e.g., 18, were created to enumerate all reachable states of the program. Such techniques provide both verification and falsification information and are very effective when the statespace of the program is finite. However, they do not scale well for programs with large state-spaces and do not apply to those with infinite state-spaces. (2) Techniques like 3[167] build an over-approximation of program behaviours, via static analysis. These techniques can handle large/infinite state-spaces, are effective for verification purposes, but are not particularly well suited for finding bugs. (3) Techniques like 24|23|4|21] explore an under-approximation of feasible program behaviours. These techniques are often inexpensive and very effective for finding bugs; they are, however, often unable to prove correctness of programs. (4) Recently, researchers have been exploring the combination of underand over-approximation by combining dynamic and static analysis techniques, respectively. Examples of this approach include [23] and the YogI project 22]. These techniques are effective both for verification and for falsification of safety

T. Touili, B. Cook, and P. Jackson (Eds.): CAV 2010, LNCS 6174, pp. 495-510, 2010.

(C) Springer-Verlag Berlin Heidelberg 2010 


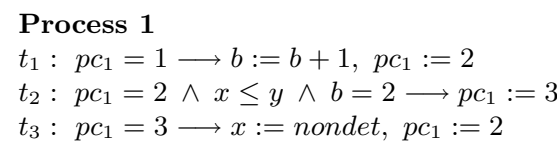

\section{Process 1}

Fig. 1. A simple two-process mutual exclusion protocol with inputs $x$ and $y$

properties but, with the exception of [23, have been limited to sequential programs 26 15]19]13. Our work fits into this category.

In this paper, we propose a novel approach for automatically checking safety properties of reactive concurrent programs (over a finite number of threads) with non-deterministic input and infinite data domains. Handling these features allows us to target programs with infinite state-spaces, uninitialized variables, and communication with an external environment (e.g., user interaction). Our approach combines symbolic execution (to deal with non-deterministic input) and predicate abstraction (to deal with infinite data domains) in an abstractionrefinement cycle. Symbolic exploration proceeds along a path until it discovers two symbolic states that match to the same abstract state - the process is called abstract matching [17. It produces an under-approximating abstract model that is more precise, in terms of feasible program behaviours it captures, than underapproximation techniques based on must transitions 24, concrete model checking and abstract matching 23, and weak reachability 4. Since we only explore feasible program behaviours, all errors we encounter are real. We then analyse the abstract model to determine if it is also an over-approximation of the reachable concrete program states. If so, we conclude safety; otherwise, we refine the abstraction, adding predicates not to remove spurious counterexamples (as in the CEGAR framework [8]) but to enable us to explore more feasible program behaviours. To our knowledge, this is the first software verification algorithm combining symbolic execution with predicate abstraction and refinement. Our contributions are thus as follows: (i) a novel method for improving precision of under-approximating models by constructing them via a combination of symbolic execution and abstract matching; (ii) a novel technique for proving safety using under-approximating models; (iii) an implementation based on 23] and an empirical evaluation comparing the two approaches.

The rest of this paper is organized as follows. In Section 2, we give a general overview of the approach, illustrating it on the example in Fig. 11 We define the notation and provide background for the remainder of the paper in Section 3 , Section 4 presents our approach in more detail, and Section 5 describes our implementation and experimental results. Section 6 compares our approach with related work. We conclude in Section 7 with the summary of our contributions and suggestions for future work.

\section{Overview}

In this section, we illustrate our approach on a simple two-process mutex protocol shown in Fig. 1. The protocol is written in a simple guarded command language. Initially, variables $x$ and $y$ are undefined (i.e., they can have an arbitrary value), 


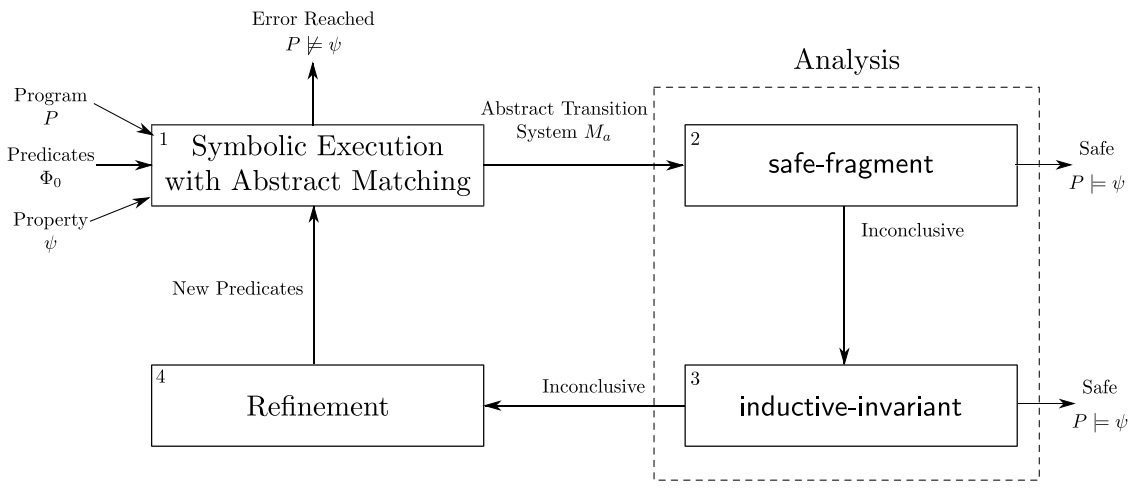

Fig. 2. Abstract analysis of symbolic executions

$b$ is $0, p c_{1}$ is 1 , and $p c_{2}$ is 1 . Process 1 starts at $p c_{1}=1$, increments $b$, and moves to $p c_{1}=2$ (transition $\left.t_{1}\right)$. At $p c_{1}=2$, it waits until $b$ becomes 2 and $x$ is less than or equal to $y$ and proceeds to its critical section at $p c_{1}=3$ (transition $t_{2}$ ). At $p c_{1}=3$, it sets $x$ non-deterministically (modelling input) and returns to $p c_{1}=2$ (transition $t_{3}$ ). Process 2 behaves analogously but uses process counter $p c_{2}$ and resets variable $y$ in its critical section. We aim to show that this protocol satisfies the mutual exclusion property: a state where $p c_{1}=3 \wedge p c_{2}=3$ is not reachable.

The high-level overview of our approach is shown in Fig. 2. To determine whether a safety property $\psi$ holds in a program $P$, we compute an abstract transition system, $M_{a}$, of $P$ w.r.t. some initial set of predicates $\Phi_{0}$ using symbolic execution with abstract matching. The state-space of $M_{a}$ is an underapproximation of reachable abstract states of $P$. If an error is found during the symbolic execution step, we report $P$ as unsafe and terminate. Otherwise, $M_{a} \models \psi$, and $M_{a}$ is passed to the analysis phase which checks, via two separate steps, whether the state-space of $M_{a}$ is also an over-approximation of $P$. If so, we are able to conclude that $P$ is safe. Otherwise, we refine the set of predicates and repeat the entire process.

Our approach follows an abstraction-refinement loop, but differs from the standard CEGAR framework [8] in two ways: (1) we compute an underapproximating abstraction of $P$ (using symbolic execution); (2) we do not rely on counterexamples to perform the refinement. In the rest of this section, we discuss each step of our approach in turn.

Symbolic Execution with Abstract Matching. Fig. 3(a) shows a symbolic execution tree of the program in Fig. 1. The initial set of predicates, $\Phi_{0}=\{x \leq$ $y, b=2\}$, consists of all the predicates from the guards of the program. A symbolic state consists of the current values of variables conjoined with the path condition that has to be satisfied in order to reach this state. In Fig. 3)(a), each state is represented as a box, with values of variables in the order $\left(p c_{1}, p c_{2}, x, y, b\right)$ appearing in the top and the path condition - in the bottom. For example, state 
$s_{1}$ is $\left(p c_{1}=1, p c_{2}=1, x=x_{0}, y=y_{0}, b=0\right) \wedge\left(x_{0} \leq y_{0}\right)$, where $x_{0}$ and $y_{0}$ are symbolic constants representing the initial value of $x$ and $y$, respectively.

We use traditional symbolic execution with one additional constraint: in each symbolic state, each predicate from $\Phi_{0}$ must be either satisfied or refuted. If necessary, we split a symbolic state by strengthening its path condition. For example, the initial state of the program in Fig. 1, $s_{0}=\left(p c_{1}=1, p c_{2}=1, x=\right.$ $\left.x_{0}, y=y_{0}, b=0\right)$, neither satisfies nor refutes the predicate $x \leq y$. Thus, it is split into states $s_{1}$ and $s_{2}$ that satisfy and refute $x \leq y$, respectively. They become the new initial states. Similarly, states $s_{5}$ and $s_{6}$ are obtained by splitting a symbolic successor of $s_{4}$. Our constraint may increase the number of symbolic states, but it ensures that each symbolic state corresponds to (or matches with) a unique valuation of all of the predicates in $\Phi_{0}$. We call such a valuation an abstract state, and define a function $\alpha(s)$ mapping a symbolic state $s$ into an abstract state.

The symbolic execution proceeds along a path until it discovers two states $s$ and $s^{\prime}$ that match the same abstract state $a$, i.e., $\alpha(s)=\alpha\left(s^{\prime}\right)=a$. For example, the symbolic path starting at $s_{1}$ and passing through $s_{3}$ is stopped at $s_{5}$. Following [23], we call this process abstract matching. Since the range of $\alpha$ is finite, symbolic execution with abstract matching is guaranteed to terminate. Of course, execution also aborts whenever it encounters an error state.

An abstract transition system $M_{a}$ is obtained from the symbolic execution tree by adding a transition between two abstract states $a$ and $a^{\prime}$ iff there is a transition between two states $s$ and $s^{\prime}$ in the symbolic execution tree, and $\alpha(s)=a$ and $\alpha\left(s^{\prime}\right)=a^{\prime}$. The abstract transition system $M_{a}$ for the execution tree in Fig. 3(a) is shown in Fig. 3 (b). In the figure, each state is a valuation to $\left(p c_{1}, p c_{2}, x \leq y, b=2\right)$. For example, $\alpha\left(s_{1}\right)=a_{1}$ and $\alpha\left(s_{2}\right)=a_{2}$. An error state is unreachable in $M_{a}$, so it is passed to the analysis phase.

Analysis: safe-fragment. This check is based on a notion of an exact transition. A transition between two abstract states $a$ and $b$ is exact iff every concrete state corresponding to $a$ can transition to a concrete state corresponding to $b$. For example, transition $a_{4} \rightarrow a_{5}$ in $M_{a}$ is exact (denoted by a solid line) whereas transition $a_{1} \rightarrow a_{3}$ in $M_{a}$ is inexact (denoted by a dotted line).

We say that a set of states $Q$, called a fragment, of an abstract transition system $M_{a}$ is exact iff (a) there is no outgoing transition from $Q$ to other states in $M_{a}$, and (b) all internal transitions within $Q$ are exact. Intuitively, all executions from concrete states corresponding to an exact fragment $Q$ are trapped in it. We say that an exact fragment $Q$ is safe iff it does not contain error states, i.e, it approximates a part of the state-space of $P$ that cannot reach an error.

safe-fragment determines whether all paths in $M_{a}$ are eventually trapped in a safe exact fragment. This is reduced to checking whether the transitions inside and between all nontrivial strongly connected components of $M_{a}$ are exact. If so, $M_{a}$ is an over-approximation of $P$ (see Section 4.2 ); therefore, none of the executions of $P$ can reach error and thus $P$ is safe. 


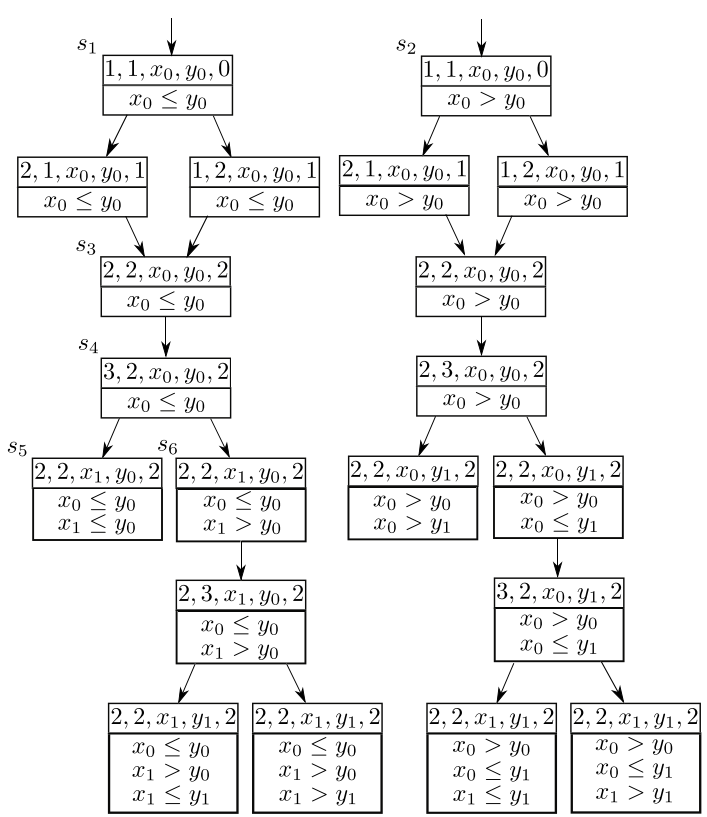

(a)

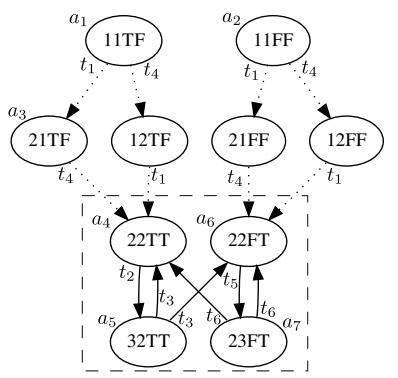

(b)

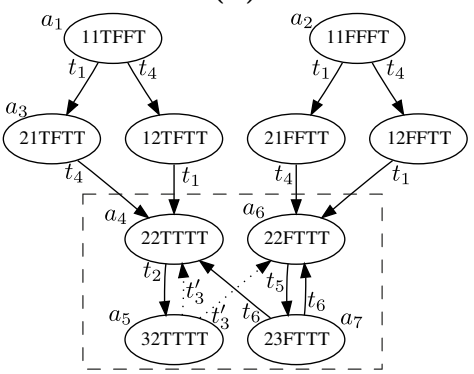

(c)

Fig. 3. (a) Symbolic execution of the program in Fig. 1] (b) its corresponding abstract transition system $M_{a} ;(\mathrm{c})$ a modified abstract transition system $M_{a}^{\prime}$

The check succeeds in our example. This is easily verified by looking at Fig. 3(b), where all paths are trapped in the safe exact fragment consisting of the states $a_{4}, a_{5}, a_{6}$, and $a_{7}$. Thus, the program in Fig. 1 satisfies the mutual exclusion property.

Analysis: inductive-invariant. This check determines whether the state-space of $M_{a}$ is an inductive invariant: i.e., it is closed under applying transitions of $P$. If so, the state-space of $M_{a}$ over-approximates that of $P$, and thus $P$ is safe. This check is complimentary to safe-fragment described above (see Section 4.2). If it fails, we move to the refinement phase.

Refinement. In this phase, we generate new predicates to refine inexact transitions of $M_{a}$. The refinement is based on computing preimage and is similar to the commonly used weakest precondition-based refinement. Although not needed in our running example, we illustrate refinement using the inexact transition $a_{1} \stackrel{t_{1}}{\longrightarrow} a_{3}$ of $M_{a}$ in Fig. 3(b). First, we compute the preimage of $a_{3}$ w.r.t. transition $t_{1}$, resulting in $\left(p c_{2}=2 \wedge x \leq y \wedge b \neq 1\right)$. Second, we add only the predicate $b \neq 1$ to $\Phi_{0}$ since program counter $p c_{2}$ is represented explicitly, and we already have $x \leq y$.

In the remainder of the paper, we formalize the above notions and evaluate the efficiency of our approach. 


\section{Preliminaries}

This section outlines the definitions and notation used in this paper.

Program. We use a guarded command language to specify programs. A program $P$ is a tuple $(V, I, T)$, where $V$ is a finite set of integer variables, $I(V)$ is an initial condition, and $T$ is a finite set of transitions. Each transition $t \in T$ is of the form $g_{t} \longrightarrow e_{t}$, where $g_{t}$ is a Boolean expression over the variables $V$, and $e_{t}$ is a set of concurrent assignments. Each assignment is of the form $x:=\operatorname{lin} E x p$ or $x:=$ nondet, where $x$ is a variable in $V, \operatorname{linExp}$ is an expression from linear arithmetic over variables in $V$, and nondet is a special expression used to denote non-deterministic input.

Transition System. A transition system over a finite set of atomic propositions $A P$ and a set of transition labels $T$ is a tuple $\left(S, R, S_{0}, L\right)$, where $S$ is a (possibly infinite) set of states, $R \subseteq S \times T \times S$ is the transition relation, $S_{0} \subseteq S$ is the set of initial states, and $L: S \rightarrow 2^{A P}$ is a labelling function, mapping each state to the set of atomic propositions that hold in it. For clarity, we write $s \stackrel{t}{\rightarrow} s^{\prime}$ to denote $R\left(s, t, s^{\prime}\right)$.

The concrete semantics of a program $P=(V, I, T)$ is a transition system $C(P)=\left(S, R, S_{0}, L\right)$ over some atomic propositions $A P$ and the set of program transitions $T$, where $S=2^{V \rightarrow \mathbb{Z}}, S_{0}=\{s \in S \mid s \models I\}$, and $s \stackrel{t}{\rightarrow} s^{\prime}$ for some $t \in T$ iff $s=g_{t}$ and $s^{\prime} \in e_{t}(s)$. By $s \models g_{t}$, we mean that the valuation of variables in $s$ satisfies the Boolean expression $g_{t}$, and $e_{t}:(V \rightarrow \mathbb{Z}) \rightarrow 2^{(V \rightarrow \mathbb{Z})}$ is a function which computes all possible states resulting from applying the assignments to some state. Finally, $L(s)=\{\phi \in A P \mid s \models \phi\}$.

Preimage and Strongest Postcondition. Let $\phi$ be a formula over program variables. The preimage of $\phi$ w.r.t. a transition $t, \operatorname{pre}(\phi, t)=\exists s^{\prime} \cdot\left(s \stackrel{t}{\rightarrow} s^{\prime} \wedge s^{\prime} \models\right.$ $\phi)$, is a formula describing the set of all states which can reach a state satisfying $\phi$ via $t$. The strongest postcondition of $\phi$ w.r.t. a transition $t, s p(\phi, t)=\exists s^{\prime} \cdot\left(s^{\prime} \stackrel{t}{\rightarrow}\right.$ $\left.s \wedge s^{\prime} \models \phi\right)$, is a formula describing the set of all states that are reachable via $t$ from a state satisfying $\phi$.

Predicate Abstraction. Let $\Phi=\left\{\phi_{1}, \cdots, \phi_{n}\right\}$ be a set of predicates over program variables. The predicate abstraction $\alpha_{\Phi}$ is a function from concrete states to Boolean formulae (abstract states) over predicates in $\Phi$. Given a concrete state $s, \alpha_{\Phi}(s)=\bigwedge_{\phi \in \Phi_{s}} \phi \wedge \bigwedge_{\phi \in \bar{\Phi}_{s}} \neg \phi$, where $\Phi_{s}=\{\phi \in \Phi \mid s \models \phi\}$ and $\bar{\Phi}_{s}=\Phi \backslash \Phi_{s}$. A concretization function $\gamma_{\Phi}$ takes a Boolean formula over $\Phi$ and returns the set of concrete states satisfying the formula. Given a Boolean formula $\psi$ over $\Phi, \gamma_{\Phi}(\psi)=\{s \in S \mid s \models \psi\}$. For a set of states $X$, we write $\alpha_{\Phi}(X)$ to mean $\bigvee\left\{\alpha_{\Phi}(s) \mid s \in X\right\}$.

A transition $a_{1} \stackrel{t}{\rightarrow} a_{2}$, where $a_{1}$ and $a_{2}$ are abstract states is a must transition iff $\forall s \in \gamma_{\Phi}\left(a_{1}\right) \cdot \exists s^{\prime} \in \gamma_{\Phi}\left(a_{2}\right)$ s.t. $s \stackrel{t}{\rightarrow} s^{\prime}$. A transition is a may transition iff $\exists s \in \gamma_{\Phi}\left(a_{1}\right) \cdot \exists s^{\prime} \in \gamma_{\Phi}\left(a_{2}\right)$ s.t. $s \stackrel{t}{\rightarrow} s^{\prime}$. In this paper, we call must transitions exact, and transitions that are may but not must - inexact. A transition $a_{1} \stackrel{t}{\rightarrow} a_{2}$ is exact iff $a_{1} \Rightarrow \operatorname{pre}\left(a_{2}, t\right)$. 
1: function $\operatorname{Refine}(P, \psi)$

$\Phi \leftarrow$ predicates from guards in $P$ and $\psi$

while true do

inductive $\leftarrow$ true

$\left(\right.$ fin, inf,$\left.A_{0}\right) \leftarrow \operatorname{SyMBOLICEXEC}(P, \Phi)$

if a state in ( $\left.\operatorname{fin}, \inf , A_{0}\right)$ satisfies $\neg \psi$ then return false

if SAFEFRAGMENT(fin, inf) then return true

symbolic execution

$A \leftarrow$ all states in (inf, fin, $\left.A_{0}\right)$

for all $\left(a_{1}, t, a_{2}\right) \in($ fin $\cup$ inf $)$ do

$\triangleright$ safe-fragment

if $\neg\left(a_{1} \Rightarrow \operatorname{pre}\left(a_{2}, t\right)\right)$ then

add predicates in $\operatorname{pre}\left(a_{2}, t\right)$ to $\Phi \quad \triangleright$ refinement

if $\neg\left(s p\left(a_{1}, t\right) \Rightarrow \bigvee A\right)$ then inductive $\leftarrow$ false

if inductive then return true

Fig. 4. Refinement loop (main function)

\section{Abstract Analysis of Symbolic Executions}

In this section, we describe our algorithm in detail and discuss its properties.

\subsection{Algorithm}

Our abstraction-refinement based verification algorithm is implemented by the function REFINE (Fig. 4) which does symbolic execution followed by safe-fragment and inductive-invariant checks and refinement (see Fig. 2). It uses two helper functions: SYMBOLICEXEC (Fig. 5), to do symbolic execution with abstract matching and to compute the explored abstract transition system, and SAFEFRAGMENT (Fig. 6), to prove safety of the abstract transition system.

REFINE initializes the set $\Phi$ with all the predicates in the program's guards and in the safety property $\psi$ (line 2), and enters the execute-analyse-refine loop (lines 3-13). It uses SYMBOLICEXEC (line 5) to compute the abstract transition system. It terminates with false if an error state is found (line 6); otherwise, it performs the safe-fragment check (line 7 ) followed, if needed, by the inductive-invariant check (lines 9-13). The Boolean variable inductive holds the result of inductiveinvariant. If it is false after inductive-invariant (line 13), then REFINE repeats symbolic execution with new predicates added to $\Phi$; otherwise, it returns true.

SYMBOLICEXEC performs depth-first symbolic execution with abstract matching. It uses the stack symbStack of sets of symbolic states to keep track of the current path. A symbolic state $s$ over a set of variables $V$ is a tuple $(f, P C)$, where $f$ is a function mapping each program variable to an integer or symbolic constant, and a path condition $P C$ is a set of constraints over symbolic and integer constants. A concrete state $c$ is represented by a symbolic state $s=(f, P C)$ iff $c \models \exists V_{s} \cdot \bigwedge_{y \in V} y=f(y) \wedge \bigwedge_{p \in P C} p$, where $V_{s}=\{z \mid \exists y \cdot f(y)=$ $z \wedge z$ is a symbolic constant $\}$. For example, the state $\left(\left\{x \mapsto x_{0}, y \mapsto y_{0}\right\},\left\{y_{0}>\right.\right.$ $\left.\left.0, x_{0}>y_{0}\right\}\right)$ denotes the set of concrete states where $y$ is strictly greater than 0 and $x$ is strictly greater than $y$.

Let $x$ be a variable in $V$ and $s=(f, P C)$ a symbolic state. The symbolic execution is done by the function ExEC (line 14 of Fig. 5) using the following rules: if $e_{t}$ is $x:=$ nondet then the result is the state $s^{\prime}=(f[x \rightarrow z], P C)$, 
$0, y>0\}$. Predicate abstraction of $s$ over $\Phi$ has two minterms: $\{x>0 \wedge y \leq$ $0, x<0 \wedge y \leq 0\}$. This leads to two new symbolic states $\left\{\left(f^{\prime}, P C^{\prime}\right),\left(f^{\prime \prime}, P C^{\prime \prime}\right)\right\}$, where $f^{\prime \prime}=f^{\prime}=\left\{x \mapsto x_{0}, y \mapsto y_{0}\right\}, P C^{\prime}=\left\{y_{0}=0, x_{0}>0,0 \leq 0\right\}$, and $P C^{\prime \prime}=\left\{y_{0}=0, x_{0} \leq 0,0 \leq 0\right\}$. Of course, tautologies like $0 \leq 0$ are discarded when the expression is simplified. This has the same worst-case complexity as the naïve approach, but allows us to use recent advances in predicate abstraction (such an AllSAT SMT solver).

In SYMBOLICEXEC, the variable trans keeps an abstraction of the path from the initial state to states at the top of symbStack as a list of tuples $(a, t)$, where $a$ is an abstract state and $t$ a transition. Whenever SYMBOLICEXEC reaches a state whose abstraction has been seen before on the current path (i.e., either it is the same as a predecessor or it appears in trans - line 16), it stops current exploration and backtracks. At this point, trans is a lasso-shaped abstract path. Functions STEM and LOOP are used to extract the transitions that occur on the stem of the path, stored in set fin and the loop of the path, stored in inf. For example, consider the symbolic execution tree shown in Fig. 3(a). When SYMBOLICEXEC takes the transition $s_{4} \stackrel{t_{3}}{\longrightarrow} s_{5}$ (corresponding to the abstract transition $a_{5} \stackrel{t_{3}}{\longrightarrow} a_{4}$ in the abstract model in Fig. 3(b)) it discovers a loop. Then, trans $=\left[\left(a_{4}, t_{2}\right),\left(a_{3}, t_{4}\right),\left(a_{1}, t_{1}\right)\right], \operatorname{sTEM}\left(\operatorname{trans},\left(a_{5}, t_{3}, a_{4}\right)\right)=$ $\left\{\left(a_{1}, t_{1}, a_{3}\right),\left(a_{3}, t_{4}, a_{4}\right)\right\}$, and LOOP $\left(\right.$ trans,$\left.\left(a_{5}, t_{3}, a_{4}\right)\right)=\left\{\left(a_{4}, t_{2}, a_{5}\right),\left(a_{5}, t_{3}, a_{4}\right)\right\}$. Note that transitions in the list trans are stored in reverse order from which they appear on the abstract path.

When SYMBOLICEXEC reaches a set of symbolic states where no transition can be taken (line 9), the transitions leading to that set of states are added to fin using the ALLPATHS function. For example, assume trans $=\left[\left(a_{2}, t_{2}\right),\left(a_{1}, t_{1}\right)\right]$ and $S=\left\{a_{3}, a_{4}\right\}$. Then, AllPaths $(S$, trans $)=\left\{\left(a_{1}, t_{1}, a_{2}\right),\left(a_{2}, t_{2}, a_{3}\right),\left(a_{2}, t_{2}, a_{4}\right)\right\}$.

SAFEFRAGMENT works by locating the fragment of the abstract model that is reached by all execution paths. This is done by finding all transitions in or reachable from inf. If all of these transitions are exact, then we conclude safety. Otherwise, we proceed to inductive-invariant.

In inductive-invariant (lines 9-13 of REFINE), for every state which is the source of an inexact transition $t$, we check if its strongest postcondition w.r.t. $t$ is a subset of the set of abstract states explored (line 12). If so, the explored abstract states over-approximate the set of reachable concrete states, and thus we can conclude safety. Otherwise, we go back to the symbolic execution stage, but now with new predicates added from preimages of destination states of inexact transitions (line 11 of REFINE).

\subsection{Soundness and Monotonicity}

Our algorithm only reports real errors. This is ensured by restricting symbolic execution to explore only symbolic states with satisfiable path constraints. Theorem 1 states that the algorithm is also sound for safety properties 2 . Of course, since property checking is undecidable, the algorithm is incomplete.

\footnotetext{
${ }^{2}$ Proofs of all theorems can be found in 1 .
} 
In the rest of this section, we represent the abstract state-space explored by SYMBOLICEXEC by a transition system $M_{a}=\left(S^{a}, R^{a}, S_{0}^{a}, L^{a}\right)$, where $A P=\Phi$, $S^{a}$ is the set of all states appearing in (fin,inf, $A_{0}$ ), $R^{a}$ is the set of all transitions appearing in fin $\cup$ inf, $S_{0}^{a}=A_{0}$, and for $x \in S^{a}, L^{a}(x)=\{\phi \in \Phi \mid x \models \phi\}$.

Theorem 1 (Soundness). Let $\psi$ be a safety property, $P$ be a program satisfying $\psi$, and $M_{c}=\left(S, R, S_{0}, L\right)$ be a transition system of P. W.l.o.g., assume that every state in $S$ is reachable from $S_{0}$. Let $M_{a}=\left(S^{a}, R^{a}, S_{0}^{a}, L^{a}\right)$ be the abstract transition system constructed by SYMBOLICEXEC in the last iteration of REFInE. Then, (i) if REFINE terminates after safe-fragment (line 7), then $M_{a}$ simulates $M_{c}$; (ii) if REFINE terminates after inductive-invariant (line 13), then $S^{a}$ over-approximates $S$ (i.e., $\left.\forall s \in S \cdot \alpha_{\Phi}(s) \in S^{a}\right)$.

As in SynERGy (see Sec. 4 in [15]), when our algorithm terminates with safefragment, the current abstraction simulates, but is not necessarily bisimular to, the concrete program. Moreover, if it terminates with inductive-invariant then the abstraction may not even simulate the concrete program.

In contrast with other under-approximating approaches, e.g., 234], our algorithm explores more states in each successive iteration than in a previous one. That is, the exploration is monotonically increasing. This ensures steady progress towards an error state (if one exists). Intuitively, we get this by keeping an abstract visited table per each path, as opposed to a unique global table as in [23].

Theorem 2 (Monotonicity). Let $\Phi$ and $\Phi^{\prime}$ be two sets of predicates s.t. $\Phi \subseteq$ $\Phi^{\prime}$. Let $P$ be a program, and $C$ and $C^{\prime}$ be the concrete states of $P$ explored by SYMBOLICEXEC under $\Phi$ and $\Phi^{\prime}$, respectively. Then, $C \subseteq C^{\prime}$.

In contrast, our approach is not monotonic for proving safety: adding new predicates may cause an exact transition used by safe-region check to become inexact 12234 . In the future, we hope to solve this problem by using an abstract domain of tri-vectors.

As discussed in Section 2, the two checks, safe-fragment and inductive-invariant, are incomparable. We prove this below.

Theorem 3. There is an abstract model $M_{a}$ constructed by SYMBOLICEXEC that passes exactly one of safe-fragment and inductive-invariant checks.

Proof. First, we give an example where safe-fragment holds but inductive-invariant fails. Consider $M_{a}$ in Fig. 3(b). Recall that it passes safe-fragment check. It fails inductive-invariant since it is not closed under strongest postcondition: $\operatorname{sp}\left(a_{1}, t_{1}\right)=$ $\left(p c_{1}=2 \wedge p c_{2}=1 \wedge x \leq y \wedge b \neq 2\right) \vee\left(p c_{1}=2 \wedge p c_{2}=1 \wedge x \leq y \wedge b=2\right)$; the second disjunct is not covered by an explored abstract state.

Second, we give an example where inductive-invariant holds but safe-fragment fails. Consider $M_{a}^{\prime}$ shown in Fig. 3(c). It is obtained from symbolically executing a program obtained by replacing transition $t_{3}$ by $t_{3}: p c_{1}=3 \longrightarrow p c_{1}:=2, x:=$ $x+1$ in the protocol in Fig. 1, and assuming that $\Phi$ includes predicates $b=0$, $b=1$, and predicates from the guards. All transitions of $M_{a}^{\prime}$, with the exceptions 


\begin{tabular}{|c|c|c|c|c|c|c|c|c|c|c|c|c|}
\hline & & \multicolumn{2}{|c|}{ Iter } & \multicolumn{2}{|c|}{ Prvr. Qurs } & \multicolumn{2}{|c|}{ Preds. } & \multicolumn{2}{|c|}{ Time(s) } & \multicolumn{2}{|c|}{ Con/Abs States } & \multirow{2}{*}{\begin{tabular}{|c|} 
Check \\
ASE
\end{tabular}} \\
\hline Program & $\psi$ & $\mathrm{ASE}$ & UR & ASE & UR & $\mathrm{ASE}$ & UR & $\mathrm{ASE}$ & UR & ASE & UR & \\
\hline bakery $_{2}$ & $\mathrm{t}$ & $\overline{3}$ & 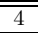 & 141 & 367 & $\overline{88}$ & 10 & $\overline{0.347}$ & 0.452 & $\overline{52 / 33}$ & 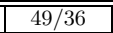 & $\overline{\mathrm{II}}$ \\
\hline RAX & $\mathrm{t}$ & 1 & - & $\overline{6}$ & - & 2 & - & 0.261 & - & $81 / 44$ & - & $\overline{\mathrm{SF}}$ \\
\hline $\mathrm{elev}_{4}$ & $\mathrm{t}$ & 1 & 4 & 418 & 5789 & 13 & 19 & 1.013 & 8.146 & $468 / 378$ & $468 / 456$ & $\mathrm{SF}$ \\
\hline elev 5 & $\mathrm{t}$ & 1 & 5 & 1169 & 26252 & 15 & 23 & 3.459 & 44 & $1256 / 910$ & $1253 / 1204$ & $\mathrm{SF}$ \\
\hline $\mathrm{elev}_{6}$ & $\mathrm{t}$ & 1 & 6 & 3156 & 105830 & 17 & 27 & 12.275 & 220.633 & $3248 / 2126$ & $3224 / 3060$ & $\mathrm{SF}$ \\
\hline $\mathrm{elev}_{7}$ & $\mathrm{t}$ & 1 & - & 7116 & - & 19 & - & 40.867 & - & $8160 / 4862$ & - & $\mathrm{SF}$ \\
\hline elev 8 & $\mathrm{t}$ & 1 & - & 15036 & - & 21 & - & 185.717 & - & $15200 / 9422$ & - & $\mathrm{SF}$ \\
\hline ticket $_{2}$ & $\mathrm{t}$ & $\overline{4}$ & 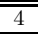 & 135 & 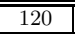 & 8 & 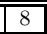 & 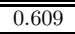 & 0.404 & $222 / 9$ & $1212 / 9$ & $\overline{\mathrm{SF}}$ \\
\hline ticket $_{3}$ & $\mathrm{t}$ & 5 & 5 & 672 & 661 & 14 & 14 & 1.413 & 0.923 & $182 / 31$ & $41 / 31$ & $\mathrm{SF}$ \\
\hline ticket $_{4}$ & $\mathrm{t}$ & 6 & 6 & 4088 & 4061 & 23 & 23 & 33.51 & 5.143 & $5011 / 129$ & $170 / 129$ & SF \\
\hline$\overline{\text { mesi }}$ & $\mathrm{t}$ & $\overline{16}$ & 16 & 6893 & 12172 & 47 & 47 & (36.61 & 49.627 & 18/18 & 18/18 & $\overline{\mathrm{SF}}$ \\
\hline berkley & $\mathrm{t}$ & 11 & 11 & 3113 & 4623 & 38 & 38 & 15.729 & 17.605 & $13 / 12$ & $13 / 12$ & SF \\
\hline b_bakery2-e & $\mathrm{f}$ & 1 & \begin{tabular}{|l|}
2 \\
\end{tabular} & $\overline{0}$ & $\overline{74}$ & $\overline{2}$ & 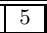 & 0.178 & 1.188 & $\overline{80 / 80}$ & $\overline{193 / 193}$ & - \\
\hline ticket $_{2}-\mathrm{e}$ & $\mathrm{f}$ & 1 & \begin{tabular}{|l|}
2 \\
\end{tabular} & 0 & 11 & 2 & 5 & 0.073 & 0.155 & $12 / 9$ & $26 / 17$ & - \\
\hline ticket $_{3}-5$ & $\mathrm{t}$ & 1 & 3 & 0 & 145 & 3 & 14 & 0.058 & 0.341 & $14 / 12$ & $93 / 81$ & - \\
\hline ticket $_{3}-10$ & $\mathrm{t}$ & 3 & 8 & 152 & 1218 & 14 & 21 & 0.525 & 2.229 & $30 / 27$ & $302 / 240$ & - \\
\hline ticket $_{3}-15$ & $\mathrm{t}$ & 8 & 13 & 1225 & 2090 & 21 & 26 & 3.107 & 5.15 & $47 / 44$ & $507 / 395$ & - \\
\hline ticket $_{3}-20$ & $\mathrm{t}$ & 13 & 18 & 2500 & 3918 & 26 & 31 & 6.869 & 9.501 & $62 / 59$ & $712 / 550$ & - \\
\hline ticket $_{3}-25$ & $\mathrm{t}$ & 18 & 23 & 3925 & 5493 & \begin{tabular}{|l|}
31 \\
\end{tabular} & 36 & 13.038 & 15.821 & $\overline{77 / 74}$ & $917 / 705$ & - \\
\hline ticket $_{3}-30$ & $\mathrm{t}$ & 23 & 28 & 5500 & 7219 & 36 & 41 & 20.762 & 34.701 & $92 / 89$ & $1112 / 860$ & - \\
\hline ticket $_{3}-35$ & $\mathrm{t}$ & 28 & 33 & 7225 & 9093 & 41 & 46 & \begin{tabular}{|l|}
46.379 \\
\end{tabular} & \begin{tabular}{|l|}
51.579 \\
\end{tabular} & $107 / 104$ & $1327 / 1015$ & - \\
\hline ticket $_{3}-40$ & $\mathrm{t}$ & 33 & 38 & 9100 & 11118 & 46 & 51 & 71.462 & 82.974 & $122 / 119$ & $1532 / 1170$ & - \\
\hline RAX-5 & $\sqrt{t}$ & 5 & 5 & $\overline{46}$ & $\overline{123}$ & $\begin{array}{ll}12 \\
\end{array}$ & 20 & $\overline{c 0.373}$ & 0.363 & 50/49 & 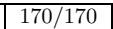 & - \\
\hline$\overline{\text { RAX-10 }}$ & $\mathrm{t}$ & 10 & 10 & 146 & 483 & 17 & 35 & 0.988 & 1.528 & $90 / 89$ & $350 / 350$ & - \\
\hline RAX-15 & $\mathrm{t}$ & 15 & 15 & 296 & 1068 & \begin{tabular}{|l|}
22 \\
\end{tabular} & 50 & \begin{tabular}{|l|}
2.031 \\
\end{tabular} & 4.341 & $130 / 129$ & $530 / 530$ & - \\
\hline RAX-20 & $\mathrm{t}$ & 20 & 20 & 496 & 1878 & 27 & 65 & 3.675 & 9.934 & $170 / 169$ & $710 / 710$ & - \\
\hline RAX-25 & $\mathrm{t}$ & 25 & 25 & 746 & 2913 & \begin{tabular}{|l|}
32 \\
\end{tabular} & 80 & 6.442 & \begin{tabular}{|l|}
19.578 \\
\end{tabular} & $210 / 209$ & $890 / 890$ & - \\
\hline RAX-30 & $\mathrm{t}$ & 30 & 30 & 1046 & 4173 & \begin{tabular}{|l|}
37 \\
\end{tabular} & 95 & 9.94 & 35.03 & $250 / 249$ & $1070 / 1070$ & - \\
\hline RAX-35 & $\mathrm{t}$ & 35 & 35 & 1396 & 5658 & 42 & 110 & 15.155 & 57.315 & $290 / 289$ & $1250 / 1250$ & - \\
\hline RAX-40 & $|\mathrm{t}|$ & 40 & 40 & 1796 & 7368 & \begin{tabular}{|l|l|}
47 \\
\end{tabular} & 125 & \begin{tabular}{|l|}
22.104 \\
\end{tabular} & 89.332 & $320 / 319$ & $1430 / 1430$ & - \\
\hline RAX-45 & 7 & 45 & 45 & 2246 & 9303 & 52 & 140 & \begin{tabular}{|l|}
30.821 \\
\end{tabular} & 133.063 & $370 / 369$ & $1610 / 1610$ & - \\
\hline
\end{tabular}

Fig. 7. Experimental results: ASE vs. UR 23

of the two transitions from $a_{5}$, are exact. safe-fragment fails on $M_{a}^{\prime}$. inductiveinvariant does not: the only interesting case is that $s p\left(a_{5}, t_{3}^{\prime}\right)=\left(p c_{1}=2 \wedge p c_{2}=\right.$ $2 \wedge b=2$ ) is covered by explored abstract states.

We have shown that our algorithm is sound and explores the concrete state-space monotonically. We have also shown that the two safety checks, safe-fragment and inductive-invariant, are incomparable. Hence, both are useful.

\section{Implementation and Experimental Results}

We have implemented our algorithm in OCaml on top of the implementation of Pasareanu et al. 23. We used GiNaC [5] for symbolic execution, MAThSAT4 [6] for computing predicate abstraction, SIMPLIFY [10] for checking exactness of transitions and computing inductive invariants, and Bradley's implementation of Cooper's method for quantifier elimination 3 . In all of our experiments, we added predicates only from those inexact transitions that are in the set inf (returned by SYMBOLICEXEC) or reachable from it.

\footnotetext{
${ }^{3}$ Available at http://theory.stanford.edu/ arbrad/sware.html
} 


\begin{tabular}{|c|c|c|c|c|c|c|c|}
\hline Program & $\psi$ & Iter. & Prvr. Qurs. & Preds. & Symb/Abs States & Time(s) & Check \\
\hline \hline ticket $_{2}$ & $\mathrm{t}$ & 4 & 523 & 12 & $5516 / 62$ & 281.134 & II \\
\hline peterson $_{2}$ & $\mathrm{t}$ & 1 & 24 & 4 & $700 / 38$ & 424 & II \\
\hline bakery $_{2}$ & $\mathrm{t}$ & 3 & 301 & 11 & $807 / 50$ & 73.965 & II \\
\hline \hline mesi & $\mathrm{t}$ & 2 & 260 & 13 & $112 / 14$ & 3.56 & II \\
\hline synapse & $\mathrm{t}$ & 2 & 62 & 7 & $34 / 9$ & 0.88 & II \\
\hline ticket $_{2}$-e & $\mathrm{f}$ & 1 & 0 & 2 & $12 / 10$ & 0.104 & - \\
\hline ticket $_{3}$-e & $\mathrm{f}$ & 1 & 0 & 3 & $10 / 10$ & 0.112 & - \\
\hline
\end{tabular}

Fig. 8. Experimental results: programs with unspecified initial states and nondeterministic input

In Fig. 17, we compare effectiveness of our abstract analysis of symbolic executions approach (referred to as ASE) with that of the under-approximation refinement algorithm of 23] (referred to as UR). We indicate whether the safety property of interest $(\psi)$ is true (t) or false (f) and report the number of iterations (Iter.), the number of theorem prover queries (Prvr. Qurs.), the total number of predicates used (Preds.), the total amount of time needed, the number of concrete and abstract states explored in the final iteration, and the check with which ASE concluded safety (II for inductive-invariant, SF for safe-fragment, and "_" when a counterexample is returned). In cases where the experiment did not finish after 15 minutes, the table entries are "-_".

Since UR can only handle a single concrete initial state and no non-deterministic input, these are the characteristics of all programs in Fig. 7. We began by checking the mutual exclusion property of the bakery protocol with two processors, where our performance is a bit better than UR. On the other hand, ASE can prove that the Remote Agent Experiment (RAX), as presented in [23, is deadlock-free in a single iteration, while UR refines indefinitely. We then verified the elevator program, elev $i$, increasing the number of floors $i$, against the property that the elevator cannot be on two separate floors at the same time. We checked mutual exclusion of the ticket ${ }_{i}$ protocol, increasing the number of processes $i$, as well as correctness cache coherence protocols mesi and berkley (these, along with their correctness properties, are taken from 9 , restricting the number of initial states to one). Our results show that ASE generally outperforms UR in terms of the number

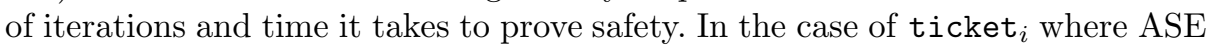
requires the same number of iterations and predicates, ASE takes more time as it explores more concrete states per iteration.

To illustrate the power of our approach at finding errors, we analysed defective versions, i.e., not satisfying mutual exclusion, of the bounded bakery $\left(\right.$ b_bakery $\left._{2}-\mathrm{e}\right)$ and ticket $\left(\right.$ ticket $\left._{2}-\mathrm{e}\right)$ protocols. We also checked whether a given ticket number $X$ in the ticket $_{i}$ protocol (ticket ${ }_{i}-X$ ) and a given counter value $X$ in the RAX example $(\operatorname{RAX}-X)$ are reachable. ASE terminates in fewer iterations than UR in the former case and in the same number of iterations but significantly fewer predicates in the latter.

In Fig. 8, we report on the results of ASE for checking properties of programs with unspecified initial states and/or non-deterministic input. Specifically, we verified mutual exclusion of ticket, where the initial ticket number is set nondeterministically, and bakery and peterson protocols, where each process stays 
in the critical section for a non-deterministic amount of time. We also verified correctness of cache coherence protocols, mesi and synapse, with undefined initial states.

In summary, ASE can analyse a wide range of programs that manipulate arbitrary integers and use non-deterministic input. And it can do so in less time, and considerably fewer iterations or with significantly fewer predicates than UR.

\section{Related Work}

The work by Pasareanu et al. [23] is the closest to ours. However, there are several key differences. First, our approach explores the state-space monotonically. Second, we use symbolic execution to deal with programs with arbitrary initial states and non-deterministic input. Third, we use over-approximation much more aggressively leading to a much faster convergence with fewer predicates. Comparison with other work is given below.

Over-approximation based techniques (e.g., 3167]) build an abstraction that has more behaviours than the concrete system and prune infeasible computations via refinement. In contrast, our refinement is based on extending the frontier of feasible program behaviours. Most of such techniques, with the exception of [7], deal with sequential programs only.

Under-approximation based techniques [23,420,24] build an abstraction that has fewer program behaviours than the concrete system. Our approach includes both reachable must and may transitions making the abstract models more precise than those that have just must transitions (e.g., 24]) and must and reverse must transitions (e.g., 4 4 ). The algorithm in 20 builds a finite bisimiluation quotient of the program under analysis, but unlike the global refinement employed by us and 23], uses a local refinement instead. We leave a comparison of the efficiency of local and global refinements for future work.

Most recent automated software verification techniques that combine dynamic analysis for detecting bugs and static analysis for proving correctness (e.g., 26 15 13 19]) concentrate on analysis of sequential programs, and unlike our approach which bounds program executions, assume terminating program executions. For example, 26] uses tests cases to explore an under-approximating abstract state-space with the hope of exploring all reachable abstract states but has no notion of refinement and thus the analysis may return false positives. Like our work, 2] uses abstraction to bound symbolic execution of programs. While this approach can handle programs with recursive data structures and arrays, its goal is debugging rather than verification, and it does not involve refinement.

[19] improves error detection capabilities of the CEGAR framework 8] by using program execution to drive abstraction-refinement. However, it does so by refining an over-approximation and is restricted to sequential programs.

Directed automated random testing (DART) [14] and its successors, [2511], run the program with random input, using path constraints to discover input that would exercise alternative program paths. The SYNERGY algorithm [15]

\footnotetext{
${ }^{4}$ see [1] for a detailed comparison with [4].
} 
combines DART-like testing with over-approximating abstractions, using results of tests to refine the abstract model and using the abstract model to drive test case generation. The end result is either a test case that reaches an error state, or an abstract model that simulates the program. Whereas DART-like approaches attempt to cover all program paths, our approach and [2326] attempt to cover all reachable abstract states. 13] presents a compositional algorithm that combines DART and over-approximating techniques. DART-like testing is used to create under-approximating (must) summaries of functions, and techniques based on [3] are used to create over-approximating (may) summaries. The authors show that alternating must and may summaries yields better results than must only or may only summaries. However, these techniques are restricted to sequential programs.

\section{Conclusion and Future Work}

We presented a novel verification algorithm that combines symbolic execution and predicate abstraction in an abstraction-refinement cycle. Our approach applies to concurrent programs with infinite data domain and non-deterministic input. Given a program and a safety property, our algorithm executes the program symbolically, while building an under-approximating abstract model. If an error is reached by symbolic execution, we terminate and report it. Otherwise, we check whether the state-space of the abstract model over-approximates all concretely reachable states. If the analysis fails, we refine with new predicates and repeat the process. Not only do we handle a much wider range of programs than related approaches, we also improve on the number of iterations and the number of predicates used, whether the property of interest is true or false.

Our current implementation is a proof of concept - more work is needed to turn it into robust verification tool that is applicable to a real programming language (such as C) with complex features (e.g., structured and recursive data types, pointers, recursion, etc.). It is also interesting to see whether the approach extends to termination (and non-termination) properties. A promising direction is to use the under-approximation to derive either a ranking function or a counterexample to termination. We leave exploring these for future work.

Acknowledgements. We would like to thank Corina Pasareanu and Radek Pelanek for giving us access to their code, the anonymous referees for their helpful comments, and the formal methods group at the University of Toronto for the fuitful discussions.

\section{References}

1. Albarghouthi, A.: Abstract Analysis via Symbolic Executions. Master's thesis, Univ. of Toronto, Dept. of Comp. Sci. (February 2010)

2. Anand, S., Pasareanu, C.S., Visser, W.: Symbolic Execution with Abstraction. STTT 11(1), 53-67 (2009) 
3. Ball, T., Rajamani, S.: The SLAM Toolkit. In: Berry, G., Comon, H., Finkel, A. (eds.) CAV 2001. LNCS, vol. 2102, pp. 260-264. Springer, Heidelberg (2001)

4. Ball, T., Kupferman, O., Yorsh, G.: Abstraction for Falsification. In: Etessami, K., Rajamani, S.K. (eds.) CAV 2005. LNCS, vol. 3576, pp. 67-81. Springer, Heidelberg (2005)

5. Bauer, C., Frink, A., Kreckel, R.: Introduction to the GiNaC Framework for Symbolic Computation with the $\mathrm{C}++$ Programming Language. J. Symbolic Computation 33, 1-12 (2002)

6. Bruttomesso, R., Cimatti, A., Franzén, A., Griggio, A., Sebastiani, R.: The MathSAT 4 SMT Solver. In: Gupta, A., Malik, S. (eds.) CAV 2008. LNCS, vol. 5123, pp. 299-303. Springer, Heidelberg (2008)

7. Chaki, S., Clarke, E., Groce, A., Jha, S., Veith, H.: Modular Verification of Software Components in C. IEEE Tran. on Soft. Eng. 30(6), 388-402 (2004)

8. Clarke, E., Grumberg, O., Jha, S., Lu, Y., Veith, H.: Counterexample-Guided Abstraction Refinement. In: Emerson, E.A., Sistla, A.P. (eds.) CAV 2000. LNCS, vol. 1855, pp. 154-169. Springer, Heidelberg (2000)

9. Delzanno, G.: Automatic Verification of Parameterized Cache Coherence Protocols. In: Emerson, E.A., Sistla, A.P. (eds.) CAV 2000. LNCS, vol. 1855, pp. 53-68. Springer, Heidelberg (2000)

10. Detlefs, D., Nelson, G., Saxe, J.: Simplify: a Theorem Prover for Program Checking. J. of the ACM 52(3), 365-473 (2005)

11. Godefroid, P.: Compositional Dynamic Test Generation. In: Proc. of POPL'07, pp. 47-54 (2007)

12. Godefroid, P., Huth, M., Jagadeesan, R.: Abstraction-based Model Checking using Modal Transition Systems. In: Larsen, K.G., Nielsen, M. (eds.) CONCUR 2001. LNCS, vol. 2154, p. 426. Springer, Heidelberg (2001)

13. Godefroid, P., Nori, A., Rajamani, S., Tetali, S.: Compositional May-Must Program Analysis: Unleashing the Power of Alternation. In: Proc. of POPL'10 (2010)

14. Godefroid, P., Klarlund, N., Sen, K.: DART: Directed Automated Random Testing. In: Proc. of PLDI'05. pp. 213-223 (2005)

15. Gulavani, B., Henzinger, T., Kannan, Y., Nori, A., Rajamani, S.: SYNERGY: a New Algorithm for Property Checking. In: Robshaw, M.J.B. (ed.) FSE 2006. LNCS, vol. 4047, pp. 117-127. Springer, Heidelberg (2006)

16. Henzinger, T., Jhala, R., Majumdar, R., Sutre, G.: Lazy Abstraction. In: Proc. of POPL'02. pp. 58-70 (January 2002)

17. Holzmann, G., Joshi, R.: Model-Driven Software Verification. In: Graf, S., Mounier, L. (eds.) SPIN 2004. LNCS, vol. 2989, pp. 76-91. Springer, Heidelberg (2004)

18. Holzmann, G.: The Model Checker SPIN. IEEE Tran. on Soft. Eng. 23(5) (1997)

19. Kroening, D., Groce, A., Clarke, E.: Counterexample Guided Abstraction Refinement via Program Execution. In: Davies, J., Schulte, W., Barnett, M. (eds.) ICFEM 2004. LNCS, vol. 3308, pp. 224-238. Springer, Heidelberg (2004)

20. Lee, D., Yannakakis, M.: Online Minimization of Transition Systems. In: Proc. of STOC'92. pp. 264-274 (1992)

21. Musuvathi, M., Qadeer, S.: CHESS: Systematic Stress Testing of Concurrent Software. In: Puebla, G. (ed.) LOPSTR 2006. LNCS, vol. 4407, pp. 15-16. Springer, Heidelberg (2007)

22. Nori, A., Rajamani, S., Tetali, S., Thakur, A.: The Yogi Project: Software Property Checking via Static Analysis and Testing. In: TACAS 2009. LNCS, vol. 5505, pp. 178-181. Springer, Heidelberg (2009) 
23. Pasareanu, C., Pelanek, R., Visser, W.: Concrete Model Checking with Abstract Matching and Refinement. In: Etessami, K., Rajamani, S.K. (eds.) CAV 2005. LNCS, vol. 3576, pp. 52-66. Springer, Heidelberg (2005)

24. Pasareanu, C., Dwyer, M., Visser, W.: Finding Feasible Counter-examples when Model Checking Abstracted Java Programs. In: Margaria, T., Yi, W. (eds.) TACAS 2001. LNCS, vol. 2031, pp. 284-298. Springer, Heidelberg (2001)

25. Sen, K., Marinov, D., Agha, G.: CUTE: A Concolic Unit Testing Engine for C. In: Proc. of ESEC/FSE'05, pp. 263-272 (2005)

26. Yorsh, G., Ball, T., Sagiv, M.: Testing, Abstraction, Theorem Proving: Better Together! In: Proc. of ISSTA'06. pp. 145-156 (2006) 